\title{
EDITORIAL
}

\section{Myocardial infarction: redefined or reinvented?}

\section{H Dargie}

Troponins have recently been promoted to playing a pivotal role in the diagnosis of acute myocardial infarction. However, a number of problems arise as a result of this "redefinition" of acute MI

T he recent joint statement by the European Society of Cardiology (ESC) and the American College of Cardiology (ACC) proposing a "redefinition" of myocardial infarction has aroused much interest but also sparked considerable controversy. ${ }^{1}$ The basis of the new definition is the promotion of troponins to a pivotal role in the diagnosis of acute myocardial infarction, coupled with the relegation of creatine kinase (CK-MB) to a secondary role; and, in the case of total CK, to the realms of history.

Elevation of troponins to centre stage in the drama of acute myocardial infarction is a direct consequence of the recent emergence of new terminology for acute coronary disease. Thus the term "acute coronary syndromes", coined to describe unstable angina and non-Q wave myocardial infarction, now also includes acute $\mathrm{Q}$ wave myocardial infarction. This has stimulated the formulation of management strategies based, at the time of presentation, on the ECG and, subsequently, on the biochemical detection of biomarkers, especially troponins. Thus patients with ST segment elevation myocardial infarction (STEMI) are candidates for thrombolysis while those without are considered to have suspected acute coronary syndromes. Those with typical ST-T changes of ischaemia are treated with low molecular heparin, aspirin, and a $\beta$ blocker. Those with less typical histories and no acute ECG changes can be observed. It is at this point that measurement of troponins has its greatest value for, if positive at 12 hours after the onset of symptoms, a diagnosis of non-ST segment myocardial infarction (nonSTEMI) can be made. Those with normal troponin values, but otherwise considered clinically to have acute ischaemia, are classified as unstable angina while patients with atypical histories can be more confidently regarded as not having ischaemic heart disease.

\section{RISK STRATIFICATION}

That raised troponin values are associated with greater cardiovascular morbidity and mortality ${ }^{2}$ indicates a potential role in risk stratification. Guidelines on the management of acute coronary syndromes without persistent ST segment elevation have been published by British, European, and US national societies ${ }^{4-7}$ and discussed. ${ }^{8}$ These vary somewhat in their endorsement of the troponin result in terms of decision making.
However, all conclude that it is a valuable index of prognosis, especially when considered in addition to the many well validated clinical indicators such as continuing evidence of ischaemia, previous myocardial infarction and other evidence of vascular disease, etc. It is salutary to note that the largest study specifically to address the issue failed to demonstrate any predictive value of a raised troponin in identifying patients who would benefit from treatment with the glycoprotein IIb/ IIIa platelet inhibitor abciximab. It is also too early to say whether a raised troponin value will have sufficiently strong independent predictive value in deciding on an invasive as compared with a conservative strategy in managing acute coronary syndromes without persistent ST segment elevation, as has been suggested in the recent TACTICS-TIMI 18 study. It is likely, however, that troponin measurement will increasingly be used to inform such decisions in conjunction with readily available clinical features.

The current, and almost universally used, World Health Organization definition" requires the presence of at least two of three criterianamely, an appropriate clinical presentation, typical ECG changes, and raised cardiac enzymes, essentially total CK or CK-MB. The ESC/ACC definition of "acute evolving or recent myocardial infarction" requires the rise and fall of a biomarker together with one of a longer list of criteria comprising ischaemic symptoms, the development of pathologic Q waves, ischaemic ECG changes, and a coronary artery intervention. For an established myocardial infarction, development of new pathologic Q waves on serial ECGs or at least two of the above four clinical features are required. The joint ESC/ACC statement also emphasises that a raised troponin concentration merely indicates cardiac damage and that other factors are necessary to describe adequately the impact of the event on cardiac function and prognosis. Thus estimating left ventricular function and detecting reversible ischaemia are welcome key recommendations in the joint ESC/ACC proposals.

Clearly, there are important differences between the two sets of criteria. According to the WHO definition, an acute myocardial infarction could be diagnosed without biochemical evidence of myocardial necrosis, while the ESC/ACC criteria mandate that one of their recommended biomarkers (preferably troponins but also CK$\mathrm{MB}$ ) be elevated and, subsequently, be shown to

Abbreviations: ACC, American College of Cardiology; CK, creatine kinase; ESC, European Society of Cardiology; STEMI, ST segment elevation myocardial infarction 
fall. This has important epidemiological consequences in patients who die too soon after admission either to have a blood test or for it to be positive.

The reasons for this redefinition have been widely discussed. Unlike CK, even CK-MB, troponins are specific to the myocyte and there is no "normal" concentration in the blood. ${ }^{8}$ Therefore, the value of troponins taken to be abnormal, $>$ 99th centile of a reference range in normal subjects, would seem to have essential biological legitimacy. Conversely, a total CK concentration of more than twice the upper limit of normal is, clearly, arbitrary.

\section{PROBLEMS OF THE NEW DEFINITION}

A number of problems arise about the adoption of the new definition. These derive mostly from the fact that its adoption would lead to many patients previously diagnosed as having unstable angina to be classified as having had a myocardial infarction, thereby, at a stroke, substantially increasing the incidence of myocardial infarction. It would also result in an overall improvement in outcome since many of these "new infarcts" will have a relatively minor degree of myocardial necrosis which should substantially improve, at least, the 30 day mortality. Clearly this would play havoc with the epidemiology $y^{9}$ and clinical management of acute myocardial infarction, for a time at least. Individual patients could be disadvantaged by being labelled as having had a heart attack or myocardial infarction in terms of employment, not to mention the adverse psychological impact the diagnosis of a myocardial infarction could cause. Current post-myocardial infarction provision would also need to be substantially increased.

Troponin measurement is undoubtedly becoming widely accepted for the triage of patients presenting with suspected acute coronary disease and can be used to rule out myocardial necrosis and rule in non-STEMI. However, the proposed extension of its role as the key determinant of the occurrence of a myocardial infarction could be regarded as a premature leap of faith rather than a logical evidence based progression.

Quite apart from the issue of overdiagnosis of myocardial infarction, there remain inexperience with and uncertainties about the robustness of the troponin assays themselves in terms of different values, not only between troponin I (TnI) and troponin $\mathrm{T}(\mathrm{TnT})$, but also among the different assays for TnI which use different antibodies. The cut points for prognostic value used in some studies used the 97.5th rather than the 99th centile for TnI of ranges of values determined from measurements in normal subjects with no evidence of coronary disease. ${ }^{10}$ Moreover, the ability of assays to meet the $10 \%$ coefficient of variability for identifying these centiles is uncertain and therefore reproducibility over time could vary.

\section{There is, therefore, little justification for adopting at face value the holistic approach suggested by the joint ESC/ACC document}

There is, therefore, little justification for adopting at face value the holistic approach suggested by the joint ESC/ACC document, at least not immediately. There has been no pilot work of any magnitude, outwith clinical trials designed for other purposes, either to inform any clinical strategy for its introduction into routine clinical practice or to investigate the problems its implementation might produce.

What is the status of these recommendations? Their adoption is not compulsory since neither the ESC nor the ACC has any mandate within the different countries of Europe, or for that matter, the USA. However, they are influential and, already, the price for the introduction of troponin measurement has been the abandonment of CK and CK-MB assays in some hospitals in the UK.

What should we advise? Practice is indeed changing and the British Cardiac Society guidelines for non-STEMI do recommend troponin assessment for risk stratification and diagnosis in acute coronary syndromes without persistent ST segment elevation. The concept of acute coronary syndromes would seem to be here to stay, and although the use of troponin was devised to cover non-STEMI and unstable angina, it is hardly surprising that its use should increase to include the whole spectrum of acute coronary disease. It would indeed be bizarre and, in the long run confusing, to use one marker, troponin, which is more sensitive and specific, for one end of a continuous spectrum of disease and a less sensitive and specific one, CK, for the other.

\section{RECOMMENDATIONS}

For the time being we recommend that cardiologists should continue to diagnose acute myocardial infarction in the accepted way until sufficient evidence emerges to justify the unqualified endorsement of a new definition. The use of troponins to exclude cardiac damage in suspected acute coronary disease should be encouraged, as should further studies of the value of troponins across the whole spectrum of acute coronary disease. Such studies should include standardisation of the two different assays and assessment of false positive elevations caused by other cardiac conditions and the confounding effect of renal failure. Comparisons of the cost effectiveness of using existing lower cost markers like total CK with the considerably more expensive troponins would seem essential. For example, what is the added value prognostically of using the troponin concentration if the total CK is already more than twice the upper limit of normal, thereby identifying a higher risk patient? Conversely, selective measurement of troponin when the CK is not significantly elevated would allow further stratification of these patients into low and higher risk subgroups. This would allow cardiologists to continue to diagnose myocardial infarction in the standard fashion in a transition period while further studies are performed to establish the true place of troponins in the overall diagnostic and prognostic algorithm for myocardial infarction.

The British Cardiac Society is in a fortunate position, having recently launched its project of myocardial infarction audit (MINAP), to have a vehicle to provide such an evaluation. MINAP provides a method of monitoring what indeed is happening throughout the country in a natural epidemiological experiment. Perhaps it could also be the catalyst in fostering appropriately designed clinical trials of practice based on the old and new criteria. Indeed, this would seem an invaluable opportunity in real life clinical epidemiology with considerable potential gains for clinical practice.

Measurement of troponins is a reflection of scientific progress and expansion in their use is inevitable. But only by adequately designed trials in the clinical situations for which they are being promoted can we learn sufficient about what they mean to recommend their widescale adoption. As with the Euro, it may be wise to wait until the conditions are right before the universal adoption of troponin measurement in the diagnosis of all cases of myocardial infarction should be recommended.

\section{REFERENCES \\ 1 The Joint European Society of Cardiology/American College of Cardiology Committee. Myocardial infarction redefined - a consensus document of the Joint European Society of Cardiology/American College of Cardiology. Eur Heart J 2000;21:1502-13. \\ 2 Antman EM, Tanasijevic M, Thompson B, et al. Cardiac specific troponin I levels to predict the risk of mortality in patients with acute coronary syndromes. N Engl J Med 1996;335:1342-9. \\ 3 Hamm CW, Ravkilde J, Gerhardt W, et al. The prognostic value of toponin T in unstable angina. N Engl J Med 1992;327:146-50. \\ 4 Bertrand ME, Simoons M, Fox KAA, et al. Management of acute coronary syndromes: acute coronary syndromes without persistent ST segment elevation. Eur Heart J 2000;21:1406-32. \\ 5 Braunwald E, Antman EM, Beasley JW, et al. ACC/AHA guidelines for the management of patients with unstable angina and non ST segment}


elevation myocardial infarction: executive summary and recommendations. J Am Coll Cardiol 2000;36:970-1062.

6 British Cardiac Society Guidelines and Medical Practice Committee and Royal College of Physicians Clinical Effectiveness and Evaluation Unit. Guideline for the management of patients with acute coronary syndromes without persistent ST segment elevation. Heart 2001:85:113-42.

7 Hamm CW, Bertrand B, Braunwald E. Acute coronary syndrome without ST elevation: implementation of new guidelines. Lancet $2001 ; 358$ : 1533-8.
8 World Health Organization. Working group on the establishment of ischemic heart disease registers. Report of the fifth working group, Copenhagen. In: Report No: Eur 8201 (5). Geneva: WHO, 1971

9 Jaffe AS, Ravkide J, Roberts R, et al. It's time for a change to troponin standard. Circulation 2000;102:1216-20.

10 Wu AH, Apple FS, Gibler B, et al. National Academy for Clinical Biochemistry Standards of Laboratory Practise: recommendations for the use of cardiac merkers in coronary artery disease. Clin Chem 1999:5:1104-21

\section{IMAGES IN CARDIOLOGY}

\section{Prosthetic valve dysfunction presenting as intermittent acute aortic regurgitation}

A

69 year old man was admitted to our hospital because of an episode of acute severe dyspnoea associated with transient loss of consciousness. His medical history revealed rheumatic aortic stenosis for which he had undergone aortic valve replacement with a No. 23 Duromedics bileaflet prosthesis in 1984. Since then, he had received adequate anticoagulant treatment and his condition had been fairly good. At admission auscultation revealed normal prosthetic valvar opening and closing clicks and a grade $2 / 6$ ejection systolic murmur. An ECG showed normal sinus rhythm with evidence of left ventricular hypertrophy. Transthoracic echocardiography was performed to evaluate prosthetic valve function. A cyclic (every 30-60 beats) failure in closure of a leaflet was seen, resulting in acute massive aortic regurgitation. In these "sporadic" cycles continuous wave Doppler (upper panel) showed the absence of closing click, followed by the signal of acute massive regurgitation (slope of the diastolic regurgitant jet $>3 \mathrm{~m} / \mathrm{s}$ ) with an increase in the velocity of forward aortic flow in subsequent systole. Colour flow showed a two dimensional display of the aortic regurgitation jet (mid panel). The patient underwent cardiac catheterisation for further evaluation. The prosthetic valve disk motion visualised by cineradiography (lower panel) showed a sporadic failure of closure of a tilting leaflet that resulted in massive aortic regurgitation. At surgery no pannus was seen and the aortic valve was replaced with a No. 21 CarpentierEdwards prosthesis. Mechanical tests revealed fatigue/structural valve deterioration of the sewing ring at the level of the pivoting mechanisms

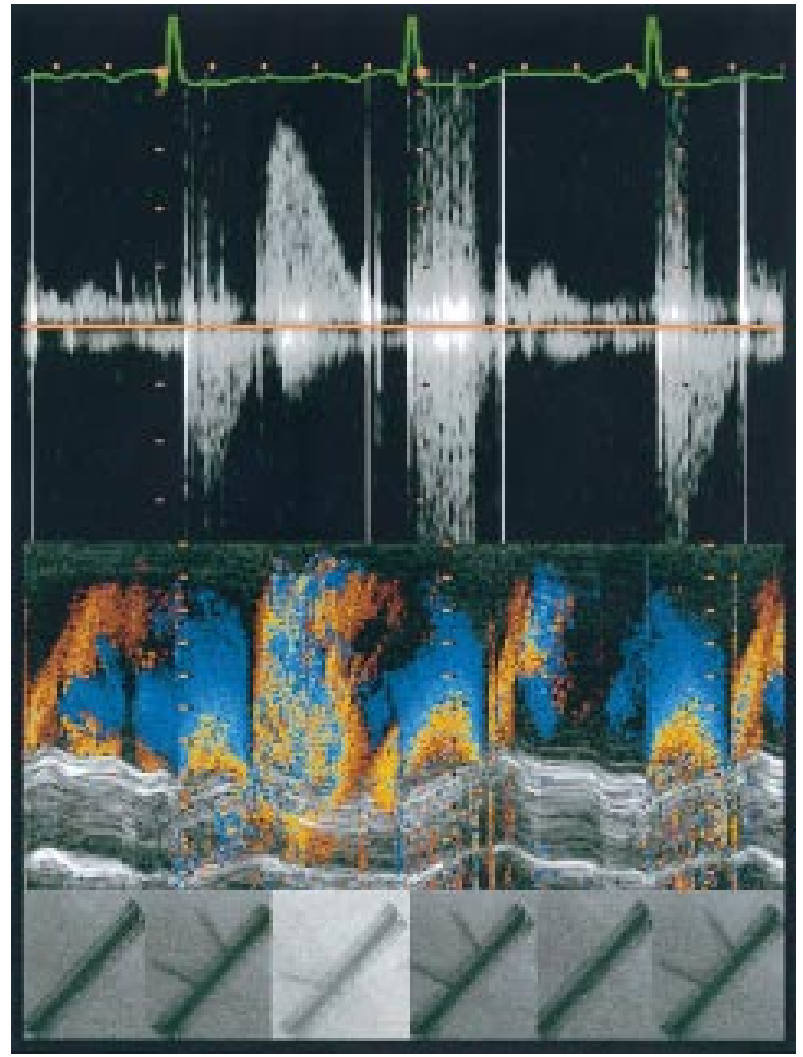

\section{STAMPS IN CARDIOLOGY}

\section{The opium poppy, morphine, and verapamil}

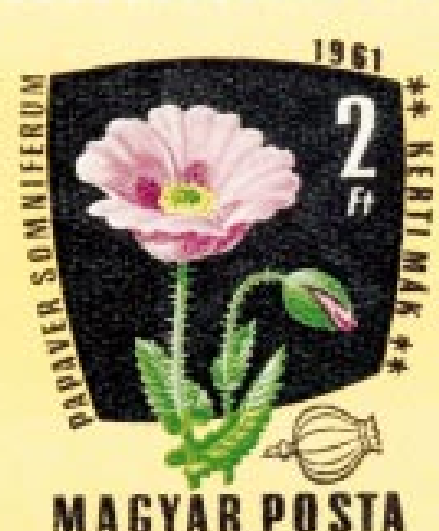

MAGYAR POSTA

Papaver somniferum, the opium poppy, has been used medicinally for over 5000 years. When the unripe seed capsule is incised a milky fluid runs out and when dried this constitutes opium (Greek opus = juice). This contains three important alkaloids-morphine, codeine, and papaverine. Morphine is unrivalled for the relief of severe pain with myocardial infarction. Before modern diuretics were introduced in 1958, it was the most valuable drug for the treatment of acute left ventricular failure. Heroin is diamorphine. Papaverine, unlike the other two alkaloids, is not an analgesic or narcotic. It acts by relaxing smooth muscle, and in animals it depresses conduction in cardiac muscle and prevents chloroform induced ventricular fibrillation. In the 1930s it was tried without success in angina, hypertension, and arrhythmias. However, it was popular for the relief of intestinal colic and this led in 1957 to the German pharmaceutical firm Knoll producing a very active synthetic analogue D365. D for their chemist, Ferdinand Dengel. This became known as verapamil and in 1963 Albrecht Fleckenstein made a seminal discovery of calcium antagonism as its mode of action. In 1972 Schamroth, Krikler, and Garrett were the first to link the arrhythmia terminating action of verapamil with the concept of calcium channel blockade.

The stamp illustrated comes from the 1961 medicinal plants set of eight issued by Hungary. Other plants featured included the blue iris, purple hollyhock, and the thorn apple. 